\title{
Introducing New Deputy Editor-in-Chief Daniel Elson, Imperial College, London, UK
}

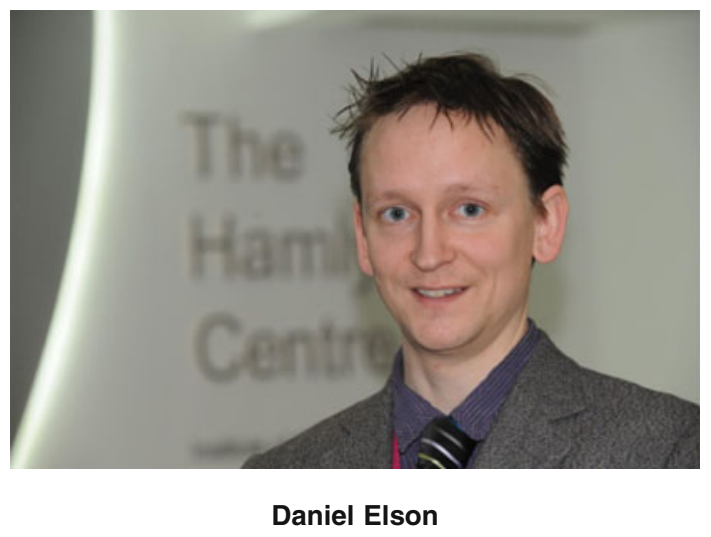

I am pleased to introduce Dr. Daniel Elson as a new Deputy Editor-in-Chief for the Annals of Biomedical Engineering. Dr. Elson joins our Editorial team that consists of myself, Dr. Scott Simon (Deputy Editor-inChief since last year), Dr. Holly Ober (Managing Editor since 2009), and our Board of Associate Editors (names and affiliations listed on the journal's masthead). Prior to this three year renewable appointment, Dr. Elson also served as an Associate Editor of the Annals for two years.

Dr. Elson obtained a first Class MSci (Hons) in Physics, Imperial College, London, followed by a Ph.D. on "Development of Ultrafast Laser Technology and its Application to Fluorescence Lifetime Imaging." He was then appointed as a lecturer in the Institute of Biomedical Engineering, Imperial College, London jointly with the Department of Biosurgery and Surgical Technology. In 2010, he joined the newly launched Hamlyn Centre for Robotic Surgery, where he is currently a senior lecturer in surgical imaging in association with the Department of Surgery and Cancer. Besides the Hamlyn Centre, Imperial College has two main centers for bioengineering: the Department of Bioengineering and the Institute of Biomedical Engineering.
The Hamlyn Centre for Robotic Surgery, Institute of Global Health Innovation is led by Professor Guang-Zhong Yang and Professor Lord Ara Darzi, and is internationally recognized for developing new surgical technologies. It is dedicated to improving the safety, cost, and quality of surgery, achieved through the unique combination of disciplines that are present within the parent departments, Department of Surgery and Cancer and Department of Computing, which are at the intersection of science, engineering, medicine, training, and healthcare policy.

Dr. Elson is leading the biophotonics and surgical imaging laboratory within the Hamlyn Centre and is working to translate promising emerging optical and biophotonic diagnostic imaging and therapy techniques into the clinic. The laboratory designs, builds, and tests optical imaging and spectroscopy instruments that are able to detect diseased tissue states using techniques such as fluorescence spectroscopy, polarization resolved endoscopy, and microconfocal endoscopy. These methods can then be used to permit minimally invasive screening procedures for the detection of various pathologies, and they promise the possibility of obtaining real-time diagnosis without the delays or tissue damage required for traditional histopathology. Beyond these optical imaging research tools, the laboratory is also developing instruments and techniques that can be used in the field of imageguided and robotic-assisted surgery.

I am thrilled to welcome Dr. Elson to our editorial team.

\author{
K. A. Athanasiou \\ Department of Biomedical Engineering \\ University of California, Davis \\ Electronic mail: athanasiou@ucdavis.edu
}

